\title{
Zusammenfassung und Fazit
}

In einem Zeitraum von insgesamt drei Jahren galt unser Forschungsinteresse den Erfahrungshorizonten von (als) Muslim:innen (Markierten), muslimischen Communities und Vertreter:innen aus Selbstorganisationen im Umgang mit dem hegemonialen Sicherheitsdiskurs in Deutschland. Wir fragten danach, wie sie auf die Anrufung als Sicherheitsgefahr antworten und interessierten uns vor allem dafür, wie es möglich ist, sich nicht gänzlich zu unterwerfen und eigene Interessen nicht aus dem Blick zu verlieren oder sie gar ins Spiel zu bringen: Wie navigieren Muslim:innen durch Strukturen des Othering und der Diskriminierung, von Ein- und Ausschlüssen, Stigmatisierung und Generalverdacht? Wie knüpfen sie an gesellschaftliche Debatten um Sicherheit an, wie beteiligen sie sich an Aushandlungsprozessen und wie positionieren sie sich, um sich Gehör für ihre Anliegen, Bedürfnisse und Erfahrungen zu verschaffen? Wie gelingt es (als) Muslim:innen (Markierten) am Diskurs teilzuhaben und dabei Definitions- und Handlungsmacht (wieder-) zu gewinnen?

Um die soziale Wirklichkeit von Muslim:innen und ihre Umgangsweisen mit dem Sicherheitsdiskurs zu rekonstruieren, bedienten wir uns zweier rekonstruktiver Methoden, die wir rassismus- und machtkritisch perspektivierten ${ }^{1}$. Ein rassismuskritischer und intersektionaler Ansatz erschien uns geboten, um - im Unterschied zur üblichen Essentialisierung und Homogenisierung von Muslim:innen - den vielstimmigen Deutungen Raum zu geben, die auf der Suche nach Strate- 
gien deren Vielfalt erst sichtbar werden lassen. Ein solcher Ansatz ist auch geboten, um Rassismus als Gesellschaft strukturierendes Machtverhältnis, das in den Wissensbeständen und Subjektivierungsweisen wirkt, im gesamten Forschungsprozess zu reflektieren und zu berücksichtigen. Den dominanzkulturellen Blick auf Muslim:innen in Rechnung stellend kehrten wir die Blickrichtung um, um muslimische Stimmen und den ihnen zugrunde liegenden Erfahrungen und Reflexionen zu hören.

Die Auswahl der Interviewpartner:innen und Diskussionsteilnehmer:innen ermöglichte es uns, eine große Bandbreite an Wissensbeständen $\mathrm{zu}$ vernehmen, die an der Schnittstelle von Alter, Gender, Sexualität, Klasse, Ability, Hautfarbe, Migration, Religion u.a. spezifisch adressiert werden. Mithilfe der Objektiven Hermeneutik ${ }^{2}$ rekonstruierten wir aus zehn Einzelinterviews Positionierungen und (Sprech-)Handlungen, während wir mithilfe der Dokumentarischen Methode $^{3}$ Aushandlungen zum Sicherheitsdiskurs in drei Gruppendiskussionen in den Blick nahmen. Die Reflexion beider Methoden in Hinblick auf ihre Methodologien und Anwendungsempfehlungen ermöglichte uns einerseits die Ausdifferenzierung von Wissensformen in hegemoniale und muslimisch/rassifizierte Wissensbestände. Andererseits konnten wir durch die Herausarbeitung der latenten Sinnstrukturen und konjunktiven Erfahrungsräume die unbewussten und die bewussten Umgangsweisen erschließen.

$\mathrm{Da}$ wir auch widerständiges (Sprach-)Handeln herausarbeiten wollten, war eine macht- und rassismuskritische Anpassung beider rekonstruktiver Forschungsmethoden nötig, um die Wissensbestände rassifizierter Personen interpretieren, analysieren und rekonstruieren zu können. Aus dem untersuchten empirischen Material ließ sich unter Berücksichtigung gouvernementalitätstheoretischer ${ }^{4}$ und rassismuskritischer Perspektiven ein Archiv an Handlungsoptionen

\footnotetext{
$2 \quad$ Wernet 2019.

3 Bohnsack 2018.

4 Foucault 2017a.
} 
nachvollziehen, mit denen Menschen mit antimuslimischer Rassismuserfahrung dem hegemonialen Sicherheitsdiskurs auf vielfältige, oft ambivalente und doch selbstermächtigende Weise begegnen.

\section{Zusammenfassung der Rekonstruktionsergebnisse}

Im Folgenden werden zunächst die Rekonstruktionsergebnisse aus den Gruppendiskussionen und Einzelinterviews vorgestellt, um anschließend allgemeine Schlussfolgerungen zu ziehen. Die vorgestellten und diskutierten Ergebnisse sind nicht repräsentativ für alle Muslim:innen und als solche Markierte. Vielmehr geben sie Aufschluss über unsere Rekonstruktion und Interpretation unterschiedlicher Deutungen und Umgangsweisen, Positionierungen und Verhandlungen, die sich unsere Gesprächspartner:innen in der Auseinandersetzung mit dem Sicherheitsdiskurs angeeignet haben und die sie in den Gesprächen mit uns und miteinander zur Sprache brachten.

\section{Ver_Handlungen}

In drei Gruppendiskussionen baten wir Muslim:innen, die sich entweder selbst als solche äußern oder als solche adressiert werden, miteinander darüber zu sprechen, wie sie den Sicherheitsdiskurs wahrnehmen, welche Auswirkungen er auf sie hat und wie sie damit umgehen. Im Gespräch mit anderen werden Reflexionsprozesse verbal zugänglich und in der Explikation und der Aushandlung von Positionen entwickelt und differenziert. Insofern boten sich Gruppendiskussionen zur Datenerhebung an.

Inter_Sektionen: Unsere Gesprächspartner:innen teilen die Erfahrung, mit kollektiven Verdächtigungen und Fragen nach ihrer Demokratiefähigkeit konfrontiert $\mathrm{zu}$ werden, sie werden alltäglich mit beleidigenden und verletzenden Begriffen als Fremde markiert und herabgewürdigt, in medialen und politischen Debatten über Sicherheit, Religion und Kultur als Bedrohung konturiert und kriminalisiert, in sicherheitspolitischer Adressierung beobachtet und 
reglementiert. Ihre Anrufung als >Muslim:innen jekte hervor ${ }^{5}$. Sie können sich dem nicht entziehen, haben aber die Möglichkeit, auf verschiedene Weisen $\mathrm{zu}$ antworten. Ihre Aussagen und Handlungen werden vor dem Hintergrund des Islam- und Sicherheitsdiskurses bewertet und reguliert. Unsere Gesprächspartner:innen antworten auf ihre Adressierung als Muslim:innen, vervielfältigen und verkomplizieren aber ihr Muslimischsein, indem sie es mit weiteren Positionierungen - Geschlecht, Sexualität, Klasse, Bildung, Migration, Kultur, Ethnizität, Sprache, Hautfarbe, Religion, Ability, Alter - kreuzen. So weisen sie einerseits die dem antimuslimischen Rassismus inhärente Essentialisierung und Homogenisierung >der Muslimin « und >des Muslim`zurück, greifen aber andererseits auf jene vergeschlechtlichten, sexualisierten, kolonialen, klassistischen Positionierungen zurück, die den antimuslimischen Rassismus im Allgemeinen und den Sicherheitsdiskurs im Besonderen durchziehen.

Ent_Solidarisierung: In allen Gruppendiskussionen wird auf das gesellschaftliche Misstrauen gegenüber Muslim:innen Bezug genommen. Der Sicherheitsdiskurs produziert ein Klima der Verdächtigung und Grenzziehung, das auch das Verhältnis innerhalb der Community berührt. Die Diskussionsteilnehmer:innen berichten davon, zu sicherheitsrelevanten Themen und Fragen Rede und Antwort stehen und sich von `bösen` Muslim:innen distanzieren und zu >liberalen`, ’konservativen zu müssen. Sie ringen um Differenzierung, handeln aus, mit wem es möglich ist, solidarisch zu sein und mit wem nicht, ohne sich selbst und die eigene Gemeinde allzu sehr in Gefahr zu bringen. So deutet sich in der Dynamik der Gespräche selbst eine gelebte Praxis der Solidarisierung und des Widerstands gegen allzu große Vereinnahmung durch die Dominanzgesellschaft an. Die Diskussionsteilnehmer:innen reflektieren Solidaritätsbekundungen, kommentieren und bewerten bisherige Erfahrungen und stellen Fragen an die Bedingungen und Chancen solidarischen Verhaltens. 
Ver_Antworten: In allen Gruppendiskussionen ziehen die Teilnehmer:innen den Staat zur Verantwortung und fordern ihn mehr oder weniger explizit auf, in demokratischen Prinzipien auch ihnen gegenüber gerecht zu werden. Sie sind sich der Anrufung bewusst, mit der sie dazu angehalten werden, ihren Beitrag zu Deradikalisierung und Prävention $\mathrm{zu}$ leisten. Sie wissen aus eigener Erfahrung, dass die Förderlandschaft auf islamistischen Extremismus fokussiert und finanzielle Anreize schafft mit dem Ziel, dass muslimische Gemeinden und Vereine die sicherheitspolitische Führung und Disziplinierung mittragen und sich selbst entsprechend führen. Sie kritisieren, auf die Rolle als Sicherheitspartner im Kampf gegen Islamismus reduziert zu werden, und dass nicht einmal in diesem Arbeitsfeld von einer Zusammenarbeit auf Augenhöhe die Rede sein kann. Stattdessen steht ihre Loyalität als Bürger:innen zur Disposition und sie werden als Muslim:innen unter Generalverdacht gestellt, sodass sie häufig mehr mit ihrer Verteidigung und Außenwahrnehmung beschäftigt sind als mit ihren community-internen Interessen und ihrem allgemeinen, gesellschaftlichen Engagement. Sie bemängeln die Intransparenz sicherheitspolitischer Apparate und Begriffsinstrumente und fordern, dass auch ihren Sicherheitsbedürfnissen und Schutzinteressen angesichts des antimuslimischen Rassismus Rechnung getragen wird.

\section{Selbst_Positionierungen}

In zehn Einzelinterviews baten wir Muslim:innen, die sich entweder selbst als solche zu Wort melden oder als solche adressiert werden, uns zu schildern, wie sie den Sicherheitsdiskurs wahrnehmen, welche Auswirkungen er auf sie hat und wie sie damit umgehen. Uns interessierte, wie sie sich angesichts ihrer Adressierung als >bedrohlich (und) fremd selbst positionieren. Um auch hier ein möglichst breites Feld an Deutungen und Praktiken zu erhalten, suchten wir unsere Gesprächspartner:innen nach ihrer Diversität aus.

Schützende Distanzierungen: Unsere Gesprächspartner:innen setzen - mehr oder weniger bewusst - verschiedene Strategien ein, um verletzenden Erfahrungen präventiv auszuweichen oder sich davon zu 
erholen. Sie gehen intellektuell auf Distanz, indem sie sich mit anderen Themen beschäftigen, sie gehen körperlich auf Distanz und wechseln die Arbeitsstelle oder den Wohnort und sie gehen emotional auf Distanz, indem sie ihre Erfahrungen verdrängen oder die überwältigenden Gefühle, die sie auslösen, zu bändigen versuchen. Sie kehren das Distanzierungsgebot gegenüber dem >islamistischen Terrorismus und >bösen Muslim:innen um, indem sie ihrerseits auf Distanz zum Bedrohungsszenario gehen. Sie erleben Rassismus als intellektuelle, physische und emotionale Herausforderung und versuchen ihr auf diesen Ebenen zu begegnen. Der Einschreibung in ihre Körper und Psychen versuchen sie durch befristete Migration, berufliche Entscheidungen und emotionale Strategien zu begegnen. Sie analysieren Rassismus in seiner Bedeutung für ihr Befinden und suchen nach Wegen, sich davon zu distanzieren, um sich in Sicherheit zu bringen.

Performative Interventionen: Unsere Gesprächspartner:innen ordnen einzelne Erfahrungen mit dem antimuslimischen Bedrohungsszenario in deren größeren gesellschaftlichen Zusammenhang ein und verweisen auf Diskursstrukturen, die sie selbst überblicken und verstehen. Sie verkomplizieren Themen, die an sie herangetragen werden, weiten Fragen aus, analysieren und kontextualisieren das eigene Sprechen. Der Diskriminierung treten sie mit selbstdefinierten Standpunkten vor dem Hintergrund eigener Erfahrungshorizonte, situierten Wissens und widerständigen Bewusstseins entgegen. Diese reichen von mimetischen Sprachhandlungen, bei denen hegemoniale Diskursfiguren angeeignet, umgedeutet und zum Zwecke der Unterbrechung des antimuslimischen Rassismus zurückgeworfen werden, über Strategien, an sie herangetragene Fragen zu sezieren, historisch-politisch zu kontextualisieren und Diskursräume auszudifferenzieren, bis hin zu aktivistischen, pädagogischen und zivilgesellschaftlichen Interventionen, bei denen hegemoniale Narrative irritiert und mit multiplen Erzählungen verkompliziert werden. Unsere Gesprächspartner:innen untergraben hegemoniale Sprechakte im antimuslimischen Bedrohungsszenario: Dessen Effekt, zum Schweigen zu bringen, zu führen und zu disziplinieren, scheitert zuweilen an performativ-analytischen Protest- 
formen, die hegemoniale Debatten umdeuten, irritieren und verkomplizieren.

Globalhistorische Verflechtungen: Unsere Interviewpartner:innen heben globalhistorische Verknüpfungen hervor und rücken vor dem Hintergrund ihrer eigenen oder familiären Erfahrungen, Narrative und Bezüge außer- und innerhalb Europas Verbindungslinien in den Fokus. Sie finden unterschiedliche Auswege, um mit der historisch unterkomplexen Narration von Bürger:inschaft im Zusammenhang mit der Anrufung als Sicherheitsgefahr umzugehen. Sie schweigen in bestimmten Gesprächssituationen, zensieren sich selbst und schützen sich, weil sie die Erfahrung machen, dass ihre Geschichte missrepräsentiert, umgedeutet und instrumentalisiert und ihre Erinnerung diskreditiert wird sowie ihre Lebensrealitäten geleugnet oder umgedeutet und sie auf das eine vermeintlich wahre Narrativ verpflichtet werden. Sie treffen Entscheidungen, wann und in welcher Form historische Adressierungen aufgegriffen und beantwortet werden, indem für sie relevante Bezüge aus dem kollektiven Gedächtnis in Erinnerung gerufen werden, sodass sie ihre eigenen Bedürfnisse im Licht der Vergangenheit und dem Zusammenspiel globalhistorischer Verflechtungen artikulieren können. Sie lesen die nationale Geschichtsschreibung gegen den Strich und bringen verdeckte Erinnerungen und Ereignisse an die Oberfläche. Sie erinnern multidirektional ${ }^{6}$, ergänzen und durchkreuzen das Feld der Erinnerung um weitere Dimensionen.

\section{Islambezogene Gouvernementalität: (Selbst-)Führung und Widerstand}

Im antimuslimischen Bedrohungsszenario tritt die Foucault'sche ${ }^{7}$ Gouvernementalität gerade dort in Erscheinung, wo weder ausschließlich Disziplin noch Strafe greifen, sondern vielmehr muslimische/muslimisierte Subjekte zur Selbstführung angerufen werden. Mit Blick

$6 \quad$ Rothberg 2009.

7 Foucault 2017a: 162. 
auf unsere Rekonstruktionsergebnisse lässt sich festhalten, dass (als) Muslim:innen (Markierte) die Erfahrung äußern, dass im hegemonialen Sicherheitsdiskurs Einfluss auf ihre Gewohnheiten und Gefühle, ihre Ängste, Erwartungen und Selbstbilder genommen wird und dass gesellschaftliche, strukturelle und institutionelle Rahmenbedingungen derart (um-)gestaltet werden, dass sich (als) Muslim:innen (Markierte) unter den hegemonialen Sicherheitsdiskurs (Salafismus, islamistischer Terrorismus, Deradikalisierung, Prävention, Leitkultur, Integration, Sicherheitspartnerschaft) $\mathrm{zu}$ fügen haben. Das bedeutet, dass in dieser neuen Regierungstechnologie Muslim:innen und als solche adressierte Menschen zusätzlich zu klassischen Machtapparaten, die kriminalisieren, pathologisieren, erziehen und ausschließen, als sehr spezifische Bevölkerungsgruppe angerufen und einbezogen werden, jedoch einseitig und instrumentell im staatlichen Interesse und vor dem Hintergrund orientalisierenden und rassifizierenden Wissens, mit dem Ziel, sie zu regulieren und Kontrolle zu dezentralisieren.

Doch Muslim:innen sind keine passiven Subjekte einer allgegenwärtigen Macht, die sie uneingeschränkt lenkt und kontrolliert, der sie sich ohnmächtig fügen oder gegenüber der sie unsichtbar bleiben. Auch muslimischen/muslimisierten Subjekten eröffnen sich durch die Unterwerfung unter den Sicherheitsdiskurs und den Namen, mit denen sie angerufen und adressiert werden, Handlungsspielräume ${ }^{8}$, sodass sie Freiheiten erweitern, eigene Positionierungen und Umgangsweisen inmitten des Spannungsverhältnisses von Macht- und Selbsttechniken selbstreflexiv, intersektional und in Ambivalenz statt Homogenität ${ }^{9}$ verhandeln.

Unsere Gesprächspartner:innen changieren zwischen verschiedenen gesellschaftlichen Positionierungen und bringen sie miteinander in Dialog. Sie berichten von (persönlichen) Rassismuserfahrungen im Bedrohungsszenario, setzen sie und den Sicherheitsdiskurs in einen politischen und historischen Zusammenhang und untermauern zugleich professionelle, wissenschaftliche und aktivistische Sprechpositionen.

$8 \quad$ Butler 2006.

9 Jain 2003; Wagenknecht 2003. 
Einige bemühen sich, antizipierten Rassismuserfahrungen vorzubeugen, zum Beispiel indem sie islamische oder als solche wahrgenommene Lektüre im öffentlichen Raum meiden, kein Arabisch sprechen oder auf deutsche Kulturgüter zurückgreifen. In der Erwartung, als Muslim:innen angesprochen und deshalb gemaßregelt oder benachteiligt zu werden, wird der öffentliche Rückgriff auf eigene Sprachen und Handlungen sowie die eigene Mobilität eingeschränkt. Das bedeutet nicht, dass unsere Gesprächspartner:innen sich den hegemonialen (Sprach-)Handlungen und den zugewiesenen Handlungsräumen vollkommen unterwerfen. Vielmehr eignen sie sich die Begrifflichkeiten subversiv an, deuten Themenfelder um, stellen Rückfragen und erweitern die Handlungsfelder um eigene Interessen, Perspektiven und Erfahrungen. Sie greifen auf performative Sprachcodes zurück, um Kontrolle über ihre Situation (wieder-) zu erlangen, indem sie Themenkomplexe sezieren und vom Gesagten abstrahieren, ihre Rassismuserfahrung problematisieren, zugleich die Situationen fachlich, wissenschaftlich und aktivistisch bewerten und untersuchen.

Den antimuslimischen Sprechakten begegnen sie mit performativen Interventionen und demonstrieren Handlungsoptionen bereits im Sprechen und Reflektieren über die Erfahrungen mit dem hegemonialen Sicherheitsdiskurs. Um sprechen zu können bzw. gehört zu werden, nehmen sie die Anrufung als Muslim:innen an, nicht aber um sie zu bestätigen, sondern um die Kategorie zu vervielfältigen und zu verkomplizieren und ihr Muslimischsein zu pluralisieren. Sie geben kollektiven wie individuellen Zuweisungen und Wirklichkeiten an der Schnittstelle von Alter, Gender, Sexualität, Klasse, Migration, Sprache, Hautfarbe, Ability und Religion Ausdruck, untergraben dadurch homogene Konstruktionen von Muslim:innen als Fremde und Andere, und stärken darüber hinaus eigene Wissensbestände, die in hegemonialen Islamund Sicherheitsdebatten marginalisiert werden.

Ingesamt wird deutlich, dass die geteilte Rassismuserfahrung Muslim:innen sowohl zu vereinen als auch zu trennen vermag. Gleichzeitig werden Selbstverständnisse und Lebensrealitäten mit anderen Erfahrungen ins Verhältnis gesetzt und zusammengeführt. In Gesprächssituationen teilen Muslim:innen verbindende und trennende Erfahrun- 
gen, sie geben einander Raum und verschaffen sich Gehör, knüpfen an eigene Erinnerungen und jene anderer aus ihren Familien oder Communities an, vervielfältigen sie oder reflektieren ausgehend davon eigene Positionierungen affirmativ oder kritisch. Sie stellen Fragen an das gesellschaftliche Zusammenleben in der Dominanzgesellschaft und unter Muslim:innen. Der Vorwurf der Illoyalität bereitet ihnen Sorgen: Sie kritisieren ein Klima der gegenseitigen Verdächtigungen und Beschuldigungen, entziehen sich Strategien des Teilens und Herrschens oder bedienen sich ihrer, und diskutieren darüber, wie eine Gesellschaft aussehen könnte, die den Bedürfnissen und Interessen aller Menschen gerecht wird, die ihre politische Teilhabe ermöglicht und CommunityStrukturen fördert und schützt, anstatt sie zu behindern, $\mathrm{zu}$ instrumentalisieren, zu stigmatisieren oder unter Generalverdacht zu stellen. Sie treten als zivilgesellschaftliche, wissenschaftliche, politische, kulturelle und soziale Akteur:innen auf, die Möglichkeiten der Solidarisierung diskutieren und Visionen austauschen für eine bessere, community-basierte Zusammenarbeit, in der sie nicht gegeneinander ausgespielt werden, sondern gemeinsame Räume gestalten, die ihnen Schutz bieten.

\section{Schlussfolgerung und Ausblick}

Den Umgang von (als) Muslim:innen (Markierten) mit dem antimuslimischen Rassismus empirisch $\mathrm{zu}$ untersuchen, legt einen methodenkritischen Perspektivenwechsel nahe. Die den Methoden zugrunde liegenden Theorien und auch ihre Anwendung müssen kritisch reflektiert und positivistische Grundannahmen aufgespürt werden, um ihren Einfluss auf den Forschungsprozess zu minimieren und der Analyse zugänglich zu machen. Hierzu sind rassismuskritische und intersektionale Zugänge in allen Phasen des Prozesses geboten: in der Formulierung der Forschungsfragestellung, im Zugang zum Feld, im Datenerhebungs- und Auswertungsprozess sowie in der Zusammensetzung des Forschungsteams und der Interpretationsgruppen. Methoden sind Werkzeuge, die machtkritisch eingesetzt auch der 
Annäherung an Wirklichkeiten und Perspektivierungen rassifizierter Menschen von Nutzen sein können. Die Reflexion grundlagentheoretischer und epistemologischer Positionen trägt zur Revision und Differenzierung von Forschungsmethoden bei.

Für unsere Studie war es notwendig, anstelle des hegemonialen >kommunikativen Wissens die sozialen Wirklichkeiten von Muslim:innen zum Ausgangspunkt der Rekonstruktion zu nehmen. Wir fragten danach, wie sie die (hegemoniale) Wirklichkeit als bedrohlich-muslimisch markierte Personen wahrnehmen, deuten und wie sie damit umgehen. Die Art und Weise, wie Muslim:innen in Forschungsvorhaben adressiert werden, welche Fragen an sie gerichtet werden, mit welchem Vokabular sie angerufen und welche Themen relevant gemacht oder in den Ausführungen der Interviewten von den Forscher:innen als relevant selektiert werden, bestimmt den weiteren Verlauf der Gespräche, der Interpretation und der abschließenden Auswertung, sodass sich durchaus auch in kritisch reflektierten Methoden stereotype Deutungsmuster wiederfinden, die bestätigt oder aufgegriffen werden.

In unserer Studie knüpfen die von uns befragten (als) Muslim:innen (Markierten) an ihrer begrifflichen und thematischen Adressierung an, weisen sie zurück oder greifen sie subversiv oder affirmativ auf, um gehört zu werden. Gerade Letzteres kann dazu führen, dass in Interviewsituationen (als) Muslim:innen (Markierte) in der Erwartung, ein bestimmtes Wissen zu präsentieren, hegemoniale Ordnungen reproduzieren oder sich an ihnen abarbeiten. In der Antizipation erneuter Rassismuserfahrungen sprechen sie in rassifizierenden Codes und unterwerfen sich - scheinbar, vorübergehend - dem Diskurs, ver-andern ihre Erfahrungen in Begriffen des hegemonialen, kommunikativen Wissens als kulturell oder religiös sfremd sie befragt, beobachtet und beforscht werden, handlungsfähig zu bleiben oder zu werden. Umso größer wirkt der Unterschied, wenn Muslim:innen sich in der Forschungssituation nicht erklären, rechtfertigen oder verteidigen und am stereotypen Wissen des Gegenübers abarbeiten müssen, sondern ein Gespräch vertiefen können, das an ihrem situierten Wissen ansetzt und nach differenzierenden Begriffen und einer Sprache sucht, mit der sie ihren Anliegen, Themen und Gefühlen 
Ausdruck verleihen können. Unsere Eingangsfrage ${ }^{10}$ eröffnete nicht nur durch die methodologische Gesprächsführung, sondern auch durch das Wissen, nicht missgedeutet, instrumentalisiert und vorgeführt zu werden, eine offene Gesprächsatmosphäre, die es möglich machte, auch sensible Themen anzusprechen und intime Perspektiven zu teilen.

Für gewöhnlich sorgt das Präfix »anti« im Begriff des antimuslimischen Rassismus für Irritation, richtet es sich doch auf den ersten Blick auf Handlungen und Diskurse, die Muslim:innen dämonisieren, ausgrenzen und kriminalisieren und sie zur Zielscheibe von Feindschaft und Gewalt machen. Eine solche Interpretation verliert aber jene Phänomene aus dem Blick, die (als) Muslim:innen (Markierte) exotisieren, paternalisieren oder - wie im Falle der Gouvernementalität - indirekt lenken, etwa indem sie als 'Sicherheitspartner angerufen und in das Othering aktiv eingebunden, zur >Leitkultur oder einem >deutschen Islam $\mathrm{zu}$ >besseren < deutschen Staatsbürger:innen zu erziehen, die islamische Religionspraxis nach christlichem Muster zu regulieren und das Verhältnis zwischen >guten und `bösen Muslim:innen weiter auszudifferenzieren. Dass auch jene Praktiken nicht im Widerspruch zur Funktionsweise des antimuslimischen Rassismus stehen, wurde in der vorliegenden empirischen Studie deutlich.

Antimuslimischer Rassismus wird als Wissensordnung wirksam, die >Muslim:innen als bedrohliche (und) fremde Andere adressiert, um ihre Ein- und Ausgrenzung zu rechtfertigen und die Barrieren und Maßnahmen, die sie an der fraglos gegebenen Zugehörigkeit hindern, $\mathrm{zu}$ legitimieren. Im Zuge dessen werden Muslim:innen als gesellschaftliche Subjekte hervorgebracht und bringen sich selbst als solche hervor. Ihre Rassifizierung hat sich freilich im Laufe der Jahrhunderte modifiziert, wie bereits Fanon ausführt. ${ }^{11}$ Im antimuslimischen Rassismus kulminieren >Religion<, >Kultur und >Herkunft< als Diskursstränge - in Intersektion mit weiteren Diskursen und Machtverhältnissen

10 Wir fragten danach, wie sie den Sicherheitsdiskurs wahrnehmen, welche Auswirkungen er auf sie hat und wie sie damit umgehen.

11 Fanon 1969: 39f. 
- und weisen >Muslim:innen $\mathrm{zu}$ - auch dann oder gerade dann, wenn sie im Inneren leben. Das konstitutive Außen wird nicht ausgeschlossen in dem Sinne, dass es entfernt wird und nicht mehr vorhanden ist, vielmehr muss es immer wieder neu nach Außen diskursiviert werden, also sichtbar bleiben als Ausgeschlossenes, sodass es das Innen weiterhin konstituieren kann. Mit Bezug auf Laclau schlägt Hall in Das verhängnisvolle Dreieck vor, zur Theoretisierung der anhaltenden Relevanz von Rassismus trotz der weit verbreiteten naturwissenschaftlichen Erkenntnis, dass es keine biologischen Rassen gibt, die Konstruktion von Rasse als "gleitenden Signifikanten « zu verstehen, der - zusammen mit Ethnizität und Nation - kulturelle Differenz legitimiert, die sich historisch und sozial entwickelt hat. Rasse, Ethnizität und Nation als Konzeptionen

»kulturelle[r] Differenz in diskursiven Termini neu zu denken [bedeutet,] Diskurs als dasjenige [zu] begreifen, was menschlichen Praktiken und Institutionen Bedeutung verleiht, was uns dazu fähig macht, die Welt zu verstehen, und folglich als das, was menschliche Praktiken zu bedeutungsvollen Praktiken macht, die genau deshalb geschichtlicher Natur sind, weil sie auf jene Weise signifizieren, auf die sie auch menschliche Differenzen markieren ${ }^{13}{ }^{13}$

Diese Theoretisierung von Rassismus unterscheidet sich von einer Deutung von Rassismus als Feindschaft oder Angst, wie sie in Begriffen der "Islamophobie« oder der »Islamfeindlichkeit« anklingen. Der antimuslimische Rassismus stellt freilich in der Geschichte des europäischen Rassismus keine Ausnahme dar, zeigt doch bereits Hall in Der Westen und der Rest ${ }^{14}$, wie zentral diskursive Aufspaltungen in sgute und >böse Andere im europäischen Expansions- und Kolonialprojekt auch in der Kolonisierung der Amerikas waren und eine intrinsische Funktion im Rassismus erfüllten. So gesehen stellt die Produktion sguter Fremder und die Bandbreite an kolonialen Strategien jenseits von Ausschluss

Laclau 2002: 67.

13 Hall 2017: 55.

14 Hall 1994. 
und Verfolgung keinen Widerspruch zum Rassismus dar, sondern eine zentrale Dimension insbesondere des postmodernen Rassismus, die rohe Formen physischer Gewalt und staatlichen Zwangs - als ultima ratio - erst denkbar macht und legitim erscheinen lässt.

Die >Anderen Macht und Herrschaft eingebunden, auch, aber nicht nur, durch Ausschluss. Wir halten daher einen Begriff des antimuslimischen Rassismus für sinnvoll, der den vormodernen und modernen Geschichten von Nationsbildung und Kolonialismus Rechnung trägt und die aktuellen Rückbezüge auf Religion, Kultur, Herkunft und Rasse (er-)neu(-t) verwebt. Es handelt sich um einen Begriff von (antimuslimischem) Rassismus, der die ambivalenten und widersprüchlichen Mechanismen nicht aus dem Blick verliert, deren Ziel und Methode es ist, nicht-weiße Menschen als Andere zu produzieren, zu disziplinieren, $\mathrm{zu}$ führen und einzuordnen. Der Begriff des Rassismus glättet die vielfältigen, gleichzeitig wirkenden und sich sowohl verstärkenden als auch irritierenden (diskursiven) Praktiken nicht zu einer einzigen Erzählung, in der Dämonisierung, Gewalt und Ausschluss als alleinige Mittel von Herrschaft und Zwang, Ausbeutung und Unterdrückung geltend gemacht werden. Rassismus dient auch und zentral der Selbstvergewisserung, sodass sowohl Aus- als auch Einschluss und sowohl »Islamophobie« als auch »Islamophilie $\ll^{15}$ dazugehören.

Im Prozess der Rassifizierung finden neben negativen durchaus romantisierende und exotisierende Vorstellungen Resonanz, die am Orientalismus anknüpfen und `Muslim:innen als Kontrastfolie zur Eigengruppe konstruieren. Ob >gut< oder >böse<, auch die zunehmenden Unter- und Zwischenkategorien halten an >Muslim:innen Figuren des ewigen Anderen fest, genauso wie an den Äquivalenz- und Differenzketten, wie Laclau und Hall sagen würden, und versuchen (als) Muslim:innen (Markierte) dazu zu bringen, von diesem Platz aus zu sprechen - oder ungehört zu bleiben.

Gleichwohl erlaubt dieses Verständnis von Rassismus (an-) zu erkennen, dass Muslim:innen auf antimuslimische Diskurse auch 
mit subversiven Gegen-Narrativen antworten, ihren Perspektiven und Stimmen widerständig Raum geben, sich vernetzen und verbünden, auch über die eigenen Communities hinaus, dass sie sich aktivistisch, künstlerisch, akademisch und zivilgesellschaftlich engagieren und mediale wie politische Debatten mitgestalten und eigenen Bedürfnissen und Interessen Gehör verschaffen. Die Performativität macht es möglich. Gleichzeitig reicht der Begriff des Widerstandes nicht aus, um der Spannbreite an Strategien Ausdruck zu verleihen, in denen Widerständiges, aber auch Affirmatives zur Geltung kommen und sowohl als das Eine als auch als das Andere gedeutet werden kann, in denen Handlungsoptionen also nicht eindeutig als Ausdruck entweder von Unterwerfung oder von Widerstand klassifiziert werden können. Vielmehr gibt es inmitten des Spektrums von Unterwerfung und Widerstand vielfältige, oft widersprüchliche Umgangsweisen, mit denen Muslim:innen innerhalb und außerhalb des diskursiven Rahmens das eigene Sprechen, die eigene Mobilität, das individuelle und kollektive Auftreten, die religiöse Praxis, das zivilgesellschaftliche Engagement, das professionelle Berufsleben und den Alltag (um-)deuten, Möglichkeiten und Grenzen austesten und so das Verhältnis von Disziplinierung und Selbstführung tagtäglich aushandeln. 
